\title{
Dual microcatheter technique for the treatment of a ruptured wide neck basilar tip aneurysm
}

\author{
Rares Filep, Dorin Nicolae Gherasim, Septimiu Popescu, \\ Botond Tokes, Lucian Marginean
}

* Department of Interventional Radiology \& Department of

Neurosurgery, Emergency County Hospital, Targu Mures, RomanIA

\begin{abstract}
Endovascular treatment is a safe and efficient therapy for intracranial aneurysms with lower complication and mortality rates compared to surgical clipping. Wide-neck aneurysms still represent a challenge to complete and safe aneurysm occlusion in spite of techniques such as stent-assisted or balloon-assisted coiling, developed in order to achieve better occlusion rates. These techniques themselves may lead to further complications, so alternative methods such as the dual microcatheter technique were developed. This technique assumes that, via two microcatheters inserted into an aneurysm, simultaneous deployment of two coils achieves a stable coil frame without the use of adjunctive devices. The aim of this paper is to present a successfully treated basilar tip wide-neck aneurysm treated with the dual microcatheter technique.

Case report. A 46-year-old male patient with acute onset of severe headache presented in the emergency room with altered state of consciousness. Nonenhanced CT scan showed subarachnoid and intraventricular haemorrhage. CT angiography revealed a wide-neck basilar tip aneurysm. Digital subtraction angiography confirmed the presence of an aneurysm with a wide, $4.9 \mathrm{~mm}$ neck.

Dual microcatheter technique was chosen as the first treatment option, while a hypercompliant balloon was kept as backup. Two microcatheters were placed inside de aneurysm and two coils were introduced in order to form a stable framing coil mass that served as a support for further coils deployed in an alternately manner through each microcatheter. No procedural complication occurred, and the patient's evolution was uneventful with no neurological deficits at discharge.

Conclusion. The dual microcatheter technique is a safe and effective therapeutic option for wide-neck ruptured or unruptured intracranial aneurysms. Periprocedural complication rates are similar to simple coiling or balloon-assisted coiling, but lower than for stent-assisted coiling.
\end{abstract}

\section{INTRODUCTION}

Endovascular treatment has become the therapy of choice for intracranial aneurysms owing to its lower complication and mortality rates, as compared to surgical clipping (5). However wide-neck aneurysms with a neck diameter greater than $4 \mathrm{~mm}$ or a dome-to-neck

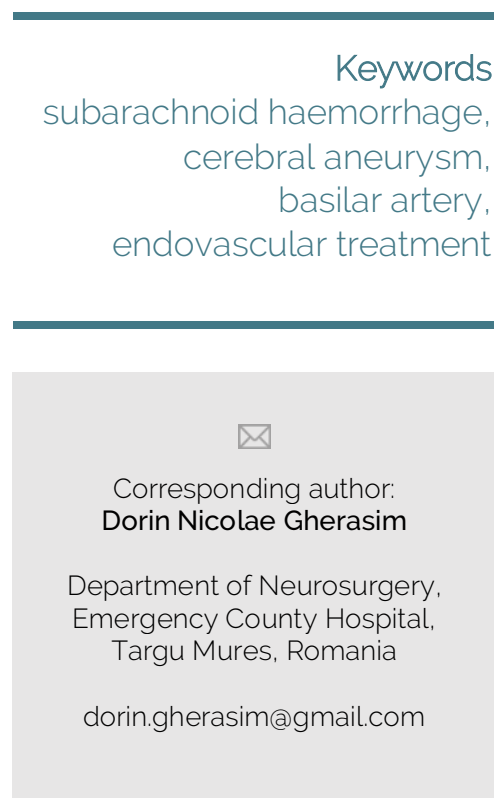

Copyright and usage. This is an Open Access article, distributed under the terms of the Creative Commons Attribution Non-Commercial No Derivatives License (https://creativecommons org/licenses/by-nc-nd/4.0/) which permits noncommercial re-use, distribution, and reproduction in any medium, provided the original work is unaltered and is properly cited.

The written permission of the Romanian Society of Neurosurgery must be obtained for commercial re-use or in order to create a derivative work.

ISSN online 2344-4959

(c) Romanian Society of Neurosurgery

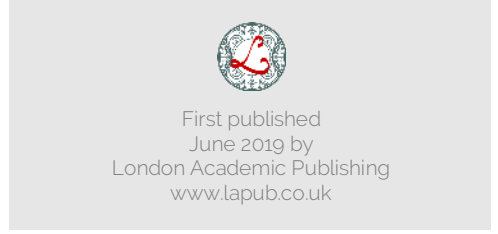


ratio less than 2 , still represent a procedural challenge attributable to the risk of intraprocedural coil herniation, a higher number of thromboembolic complications and higher rate of long-term recanalization (6).

Different techniques have been developed to assist wide-neck aneurysm coiling by covering the neck during coil deployment, thus allowing denser packing and better long-term occlusion rates. Stents and balloons have been used for this purpose, but both techniques have their limitations. Stent placement might be difficult in tortuous vessel anatomy and is associated with delayed thromboembolic events and in-stent stenosis. Balloon-assisted coiling (BAC) requires temporary flow arrest and may be associated with increased risk of vessel rupture and thromboembolic complications (7).

One alternative to the above-mentioned procedures is a less used and under-reported method, the dual microcatheter technique (DMT), which requires simultaneous placement of two microcatheters in the aneurysm sac and deployment of coils through both of them with the aim of achieving a stable coil frame without the use of adjunctive devices (1).

The aim of this paper is to present the case of a patient with a wide-neck basilar tip ruptured aneurysm, successfully treated using the DMT and to discuss its advantages and disadvantages compared to other techniques used for wide-neck aneurysm coiling.

\section{CASE REPORT}

A 46-year-old male patient presented in the emergency department with abrupt-onset severe headache. Clinical examination revealed a drowsy and confused patient (Hunt-Hess 3). Non-enhanced CT and CT angiography of the head performed on 64channel machine (Siemens, Erlangen, Germany) showed a subarachnoid and intraventricular hemorrhage (mFisher 4) and a wide-neck basilar tip aneurysm. (FIGURE 1: a, b, c)

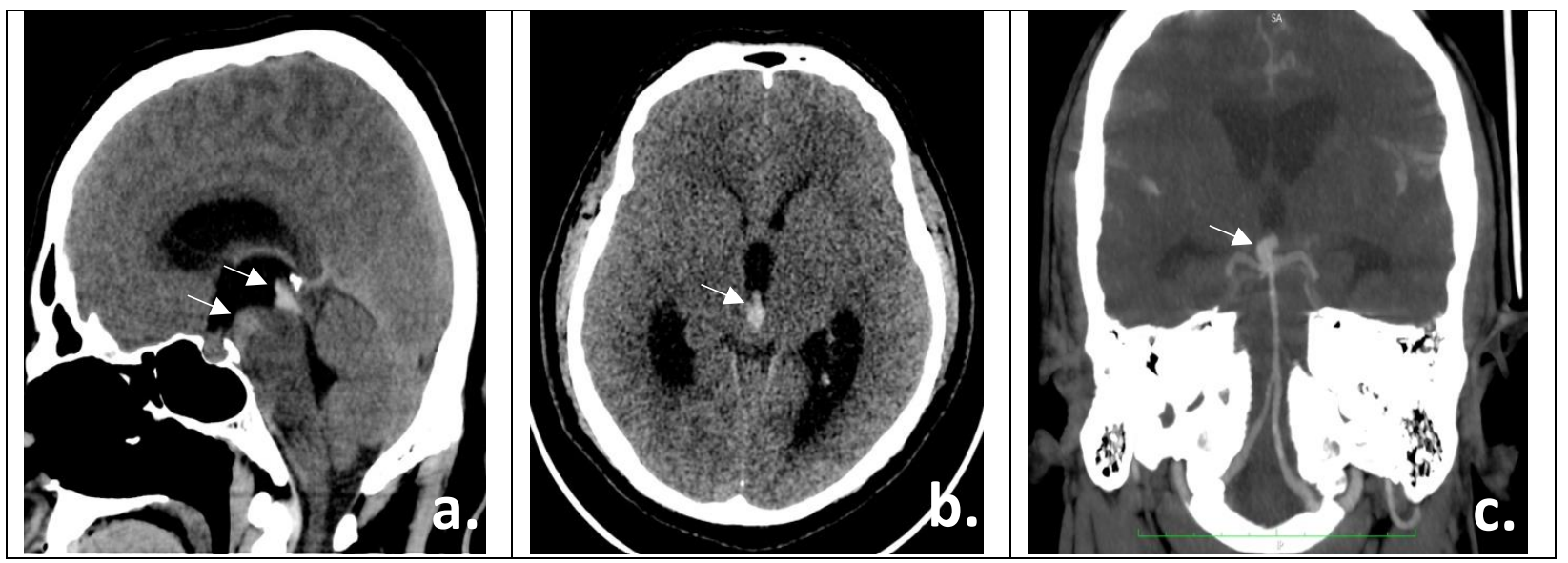

FIGURE 1. a. Sagittal NCCT image reveals a small amount of subarachnoid blood in the interpeduncular cistern and the $3^{\text {rd }}$ ventricle (white arrows); b. Axial NCCT slice shows the blood clot in the $3^{\text {rd }}$ ventricle (white arrow); c. Coronal CTA image reveals the basilar bifurcation aneurysm (white arrow)

NCCT = non-contrast computed tomography; CTA = computed tomography angiography

Digital subtraction angiography (DSA) and 3D rotational angiogram obtained on a Siemens biplane system (Siemens, Erlangen, Germany) showed the basilar tip aneurysm measuring 7.1/5.1/5.4 mm (CC/LL/AP diameters) with a neck of $4.9 \mathrm{~mm}$ (dometo-neck ratio of 1.4), and a small secondary lobule along the left superior border of the aneurysm, thought to be the site of bleeding (FIGURE 2: $a, b$ ).
Under general anesthesia the right common femoral artery was accessed with a 6F Cordis sheath (Cordis, Freemont, California) and a 6F Chaperon guiding catheter (Terumo, Tokyo, Japan) was placed in the right vertebral artery (VA). A Headway 17 microcatheter (Terumo, Tokyo, Japan) was then advanced into the aneurysm and a Target 360 standard $5 \mathrm{~mm} \times 15 \mathrm{~cm}$ coil (Stryker, Michigan, USA) 
was deployed. At this point an intravenous bolus of 2500 units of heparin was administered. The coil was quite stable in the aneurysm sac without impingement on the basilar artery. (FIGURE 2: c.) We kept it attached to its introducer wire nevertheless, with the idea to keep it as support for further coils that will be inserted through a second microcatheter.

A $5 F$ Cordis femoral sheath was inserted on the opposite side and a second Headway 17 microcatheter was introduced into the aneurysm via a 5F Navien support catheter (Medtronic, Minneapolis, USA) placed in the left VA. A 3D Axium Prime $5 \mathrm{~mm} \times 10 \mathrm{~cm}$ coil (Medtronic, Minneapolis, USA) was introduced through the second microcatheter, and by intertwining with the first coil, formed a secure, stable coil mass throughout the entire volume of the aneurysm. (FIGURE 2: $d$, e) The second coil was detached, and an additional bolus of 2500 IU of heparin was administered. A third HydroCoil $3 \mathrm{~mm} \times 8 \mathrm{~cm}$ coil (Microvention, California, USA) was introduced through the second microcatheter, followed by the careful detachment of the very first coil. Three further hydrogel-coated coils were introduced through both microcatheters, alternately, resulting in satisfactory occlusion of the aneurysm judged by the Raymond-Roy occlusion classification as 1 (FIGURE 2: f). The patient had an uneventful postoperative course and was discharged 2 weeks later without neurological deficits, with a modified Rankin scale 0.

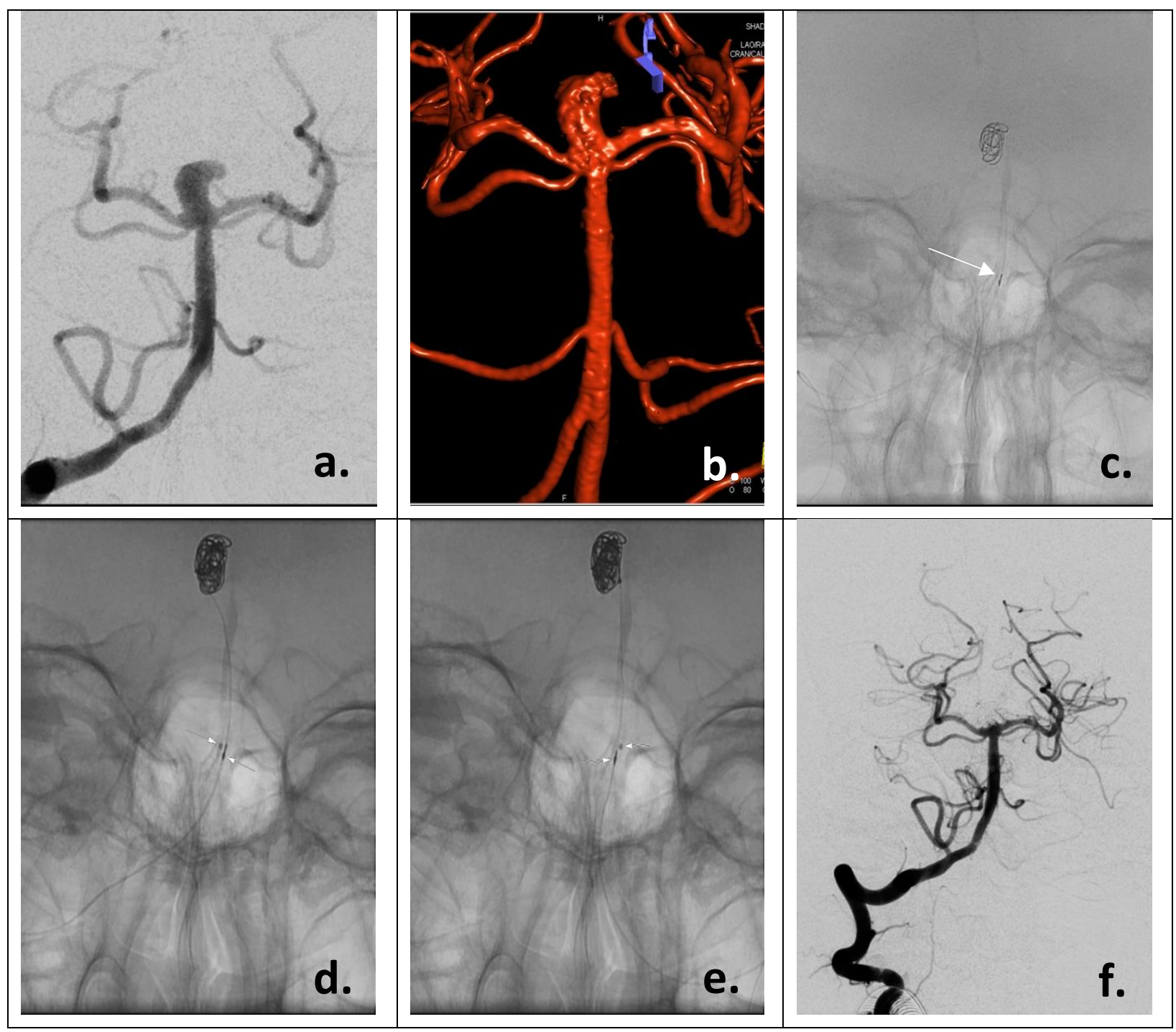

FIGURE 2. a. AP DSA image from a right VA injection shows a basilar tip aneurysm; b. 3D rotational angiographic reconstruction; $\mathbf{c}$. AP unsubtracted image shows the first microcatheter and the first coil inside the aneurysm (white arrow); $\mathbf{d}$. AP unsubtracted image 
reveals the second microcatheter (white arrows) and a further coil inserted into the aneurysm; e. Further coils are deployed simultaneous through both of the microcatheters; $\mathbf{f}$. Final control DSA angiogram shows the complete obliteration of the aneurysm. $\mathrm{AP}=$ anteroposterior, $\mathrm{DSA}=$ digital subtraction angiography

\section{DISCUSSIONS}

Since its introduction in 1998 by Baxter's group (4), DMT has been used as an alternative to balloon or stent-assisted coiling (SAC) for wide-neck intracranial aneurysms. It involves the use of two microcatheters inserted in the aneurysm sac followed by simultaneous or sequential deployment of coils. The general principle behind this technique is the stability obtained by the "entanglement" of the first two framing coils inserted simultaneously, thus creating a larger coil frame that prevents impingement on or herniation of the coil loops into the parent artery. One of the coils is usually detached at the end of the procedure thus conferring additional stability for further coils.

The technique is aimed at wide-neck saccular aneurysms with a dome-to-neck ratio less than 2 , irregular aneurysms with multiple daughter sacs and complex configurations and aneurysms with a vessel emerging from the neck (5). We considered DMT as the first therapeutic option for our case while a balloon was kept as back-up in case the coils were not stable inside the aneurysm. SAC was excluded because the aneurysm was ruptured, and dual antiplatelet therapy was necessary. For a more detailed description of the technique we propose a step-by-step guide on how to approach aneurysms that are amenable to treatment with this procedure.

\section{General procedural considerations}

Endovascular treatment of intracranial aneurysms should be performed with the patient under general anesthesia. In our institution all catheters are continuously flushed with saline containing heparin ( $2500 \mathrm{IU}$ heparin in $500 \mathrm{ml}$ of saline). Our protocol is to administer a bolus of 5000 IU heparin intravenously after the deployment of the first coil, although during this procedure, 2500 IU were administered after insertion of both guiding catheters in order to prevent thromboembolic events, and $2500 \mathrm{IU}$ following the deployment of the first coil.

\section{Femoral artery access and guiding-catheter (GC) choice.}

Uni- or bilateral access depends on how many GC's are needed. Generally, the dual microcatheter technique can be safely performed through one GC. In the majority of cases the internal carotid arteries (ICA) or at least one of the vertebral arteries (VA) can easily accommodate one larger device, although cases with small VA's may require the use of two GC's in cases with posterior circulation aneurysms (4). Advantages of using a unilateral approach are the smaller complication rates related to femoral artery puncture and large vessel access, like arterial spasm, dissection and embolic events. Theoretically, using two GC's can lead to a two-fold increase in such complications. The use of two GC's can be avoided because the majority of 6 or 7 French (F) devices have a large enough lumen to contain both microcatheters. Disadvantages of using only one device are the difficulty of obtaining quality roadmaps or control angiograms with two microcatheters that occupy a large luminal area of a $6 \mathrm{~F} \mathrm{GC}$, although this can be overcome with the use of a 7F device. Another disadvantage of two microcatheters inserted through the same GC is related to the risk of inadvertent forward/backward movement while manipulating them, which can lead to aneurysm perforation or dislodgement of the coil mass $(4,3)$. Due to the limited experience with this technique we felt safer with a bilateral approach in order to avoid inadvertent microcatheter movement and to have access with a hypercompliant balloon if the coils were not stable or if rupture of the aneurysm occurred.

\section{Aneurysm access}

The first option is to try coiling the aneurysm with one microcatheter. If this fails due to coil herniation or impingement on the parent artery, the second microcatheter is positioned as follows. To anticipate the deployment behavior of the coils it is useful to divide the aneurysm in two imaginary compartments, each one occupied by one of the first two coils, a simple example is to have a proximal and a distal compartment, and to position the tip of the two microcatheters in the corresponding compartment (3).

\section{Coil selection, deployment and detachment}

Coil choice depends on the configuration of the aneurysm. For irregular aneurysms with multiple 
daughter sacs soft coils are recommended to avoid perforation. If this is not the case, standard, complex coils are preferred due to their larger radial force providing a better "anchoring" inside the aneurysm. The first coils should be longer, thus minimizing the number of subsequent coils necessary for a dense final packing. After achieving a stable frame with the first two coils, one of them is detached and further coils are inserted until complete obliteration is obtained, while the other is left attached to its pusher-wire. The decision which coil to detach first is usually based on its stability: the more stable looking coil is to be detached first, because it provides a better scaffold for further coils. Another consideration to which coil should be detached first is the position of the microcatheter tip. It is safer to insert coils through the catheter sitting closer to the neck of the aneurysm than through the one positioned deeper inside due to the risk posed by the deep deposition of coils that might "push" the whole coil construct outside and into the parent vessel, consequently the one sitting deeper should be detached earlier $(1,3,4)$.

\section{Microcatheter removal}

A potential risk of retrieving the microcatheters at the end of the procedure is the extraction of the coil mass outside the aneurysm. The best way to avoid this if the microcatheter's tip is deep inside the aneurysm, is to push gently on the pusher-wire of the last coil until the tip protrudes outside, and only afterwards to detach the coil (6).

DMT is a relatively under-used method for intracranial aneurysms compared to balloon or stent-assisted coiling, with only few case reports, series and retrospective studies published in the literature so far. The safety and efficiency of this approach has been assessed by Durst et al in their retrospective analysis of 100 wide-necked aneurysms (2). They reported a morbidity and mortality of $1 \%$ and $2 \%$ respectively. Intraprocedural rupture occurred in 3 patients (3\%), 2 were successfully stopped immediately and 1 patient died due to massive hemorrhage and hydrocephalus. One other patient died as a consequence of the coils herniating in the parent vessel, obliterating the lumen and subsequent ischemic stroke. DMT was successfully carried out in 91 cases (91\%). The remaining 9 failed due to the impossibility of stabilizing the coils inside the aneurysm.
Retreatment was necessary in $18 \%$ of cases after recanalization (2).

The relatively low major complication rate of DMT (3\%) is similar to that reported for simple coiling (0.6$5.1 \%)$ or BAC (0.9-3.8\%), but much lower than for stent-assisted coiling (SAC) (9.4-12.2\%) (7). Two common downsides of BAC and SAC are the greater experience needed for device manipulation and the specific risks imparted by these devices on the procedure. Balloons add the risk of intraprocedural vessel rupture while stents are permanent intraluminal devices prone to in-stent stenosis and thrombosis, requiring long-term antiplatelet therapy with an additional hemorrhagic risk. Conversely, no special training is needed for DMT since the only devices used are those required for simple coiling, although special care is mandatory during aneurysm access, because two catheterization procedures mean an increased risk of wall perforation with either the guidewire or the microcatheters.

One major issue of DMT is the retreatment rate of $18 \%(7)$, that compared to BAC, between $5.7 \%$ and $15.6 \%$, and SAC, between $4.3 \%$ and $13.3 \%$ (8) is relatively high. This is especially important for young patients due to their longer life expectancy, therefore a judicious use of DMT is necessary in this patient population.

\section{CONCLUSIONS}

The dual microcatheter technique is a safe and effective therapeutic option for wide-neck ruptured or unruptured intracranial aneurysms. Periprocedural complication rates are similar to simple coiling or balloon-assisted coiling, but lower than for stent-assisted coiling.

\section{REFERENCES}

1. Baxter BW, Rosso D, Lownie SP. Double microcatheter technique for detachable coil treatment of large, widenecked intracranial aneurysms. AJNR Am J Neuroradiol. 1998 Jun;19(6):1176-8.

2. Durst CR, Starke RM, Gaughen JR, Geraghty S, Kreitel KD, Medel $R$, et al. Single-center experience with a dual microcatheter technique for the endovascular treatment of wide-necked aneurysms. J Neurosurg. American Association of Neurological Surgeons; 2014 Nov. 121 (5): 1093-101.

3. Horowitz M, Gupta R, Jovin T. The dual catheter technique for coiling of wide-necked cerebral aneurysms. An underreported method. Interv Neuroradiol. 2005 Jun 30; 11 (2):155-60. 
4. Kwon O-K, Kim SH, Kwon BJ, Kang H-S, Kim JH, Oh CW, et al. Endovascular treatment of wide-necked aneurysms by using two microcatheters: techniques and outcomes in 25 patients. AJNR Am J Neuroradiol. 2005 Apr;26(4):894-900.

5. Molyneux AJ, Kerr RS, Yu L-M, Clarke M, Sneade M, Yarnold JA, et al. International subarachnoid aneurysm trial (ISAT) of neurosurgical clipping versus endovascular coiling in 2143 patients with ruptured intracranial aneurysms: a randomised comparison of effects on survival, dependency, seizures, rebleeding, subgroups, and aneurysm occlusion. The Lancet. 2005 Sep;366(9488):80917.
6. Pierot L, Biondi A. Endovascular techniques for the management of wide-neck intracranial bifurcation aneurysms: A critical review of the literature. Journal of Neuroradiology. Elsevier Masson SAS; 2016 Mar 11;:1-9.

7. Piotin M, Blanc R. Balloons and stents in the endovascular treatment of cerebral aneurysms: vascular anatomy remodeled. Front Neurol. Frontiers; 2014;5(Suppl 3):41.

8. Wang $F$, Chen $X$, Wang $Y$., Bai $P$, Wang $H-Z$, Sun $T$, et al. Stent-assisted coiling and balloon-assisted coiling in the management of intracranial aneurysms: A systematic review \& meta-analysis. J Neurol Sci. 2016 May 15; 364: $160-6$. 